\title{
Numerical Analysis of the Strength of the Wet Steam Turbine Blade
}

\author{
Bing Guo ${ }^{1,2,3}$, Weixiao Tang ${ }^{1,2,3, a}$, Jin Liu ${ }^{1,2,3}$ and Tianhui Zhen ${ }^{1.2,3}$ \\ 1 School of Mechanical Engineering, Shandong University, Jinan, 250061, P.R. China \\ ${ }^{2}$ Key Laboratory of High Efficiency and Clean Mechanical Manufacture, Ministry of Education, Jinan, P.R. China \\ ${ }^{3}$ National Demonstration Center for Experimental Mechanical Engineering Education, Jinan, P.R. China
}

\begin{abstract}
A method to examine the influences of inlet wetness fraction and inlet angle on the strength performance of the rotating blade considering the effects of wet steam non-equilibrium condensation was proposed. A wildly verified numerical model was employed to simulate the wet steam flow to obtain the pressure distribution on the blade surface. Based on the finite element method, the strength analysis was carried out. Results show that the von-Mises stress of the rotor blade would increase with the inlet wetness fraction and the inlet angle. The sensitive range of studied inlet condition is located at $A_{\text {in }} \in[0,15], W_{\text {in }} \in[0.03,0.04]$, in which the increases of the von-Mises stress are over $40 \%$.
\end{abstract}

\section{Introduction}

Excessive stress may shorten the effective life of the rotating blades and threaten the safety of turbomachinery. Especially for the turbine operating on wet steam, such as nuclear turbines and the last stage of low pressure gas turbines, in which the non-equilibrium condensation (NEC) process of the wet steam may greatly enhance the extent of flow non-uniformity to generate more intense fluid excitation and induce higher stress of the rotating blade. Hence, it is of great practical interest in the design of wet steam turbines to predict the strength performance of the rotating blades considering the effects of the NEC.

Currently, research on the fluid excitation generate from the NEC of wet steam is still insufficient. Generally, the condensation of wet steam is regarded as an equilibrium process, either or the condensation is ignored and the wet steam is simplified as an ideal gas. After above simplifications, the vortex shedding from the upstream blade is regarded as the major fluid excitation, i.e. the wake excitation. A lot of studies about the wake excitation and its induced vibration have been carried out, to name a few, Lau [1] used the Karman vortex street (KVS) to model the wake excitation and analyzed the vortex-induced vibrations of a 2D airfoil. García [2] numerically calculated the wake excitation acting on the rotor blade of the last stage of $110 \mathrm{MW}$ steam turbine. However, the strength performance of the rotating blade considering the effects of NEC hasn't been well clarified due to the difficulty in calculating NEC-induced excitation.

In this paper, the effects of wet steam NEC on the strength performance of the rotating blade are numerically studied. In which the NEC-induced excitation (pressure distribution on the blade surface

\footnotetext{
${ }^{a}$ Corresponding author : tangwx@sdu.edu.cn
} 
during NEC process) can be obtained from the numerical simulation of the wet steam flow field based on the non-isothermal nucleation theory and the droplet growth model [3-5] that implemented in ANSYS CFX. Once the NEC-induced excitation is obtained, the strength performance of the rotating blade can then be analyzed use the finite element method (FEM) through the strength calculation under the pressure load with commercial software ANSYS Workbench. Besides, the influences of the wet steam inlet conditions (intake angle and wetness fraction) are analyzed.

\section{Wet steam model}

The effects of NEC on the strength performance of the rotating blade are determined through the pressure distribution on the blade surface during NEC process. To obtain the pressure distribution, a NEC model of wet steam is necessary. The wet steam is a mixture of two-phases, in which the primary phase is the gaseous-phase consisting of water-vapor (denoted by the subscript $v$ ), the secondary phase is the liquid-phase consisting of condensed-water droplets (denoted by the subscript l).

The numerical calculations can be carried out based on a compressible Navier-Stokes equations using Eulerian-Eulerian approach to govern the condensing flow. The equations are given by [6]

$$
\begin{gathered}
\frac{\partial}{\partial t}\left(\begin{array}{c}
\rho \\
\rho V_{i} \\
\rho H
\end{array}\right)+\frac{\partial}{\partial x_{i}}\left(\begin{array}{c}
\rho V_{i} \\
\rho V_{i} V_{j}+p-\mu_{t} \frac{\partial V_{i}}{\partial x_{j}} \\
\rho H+p-\lambda_{t} \frac{\partial T_{v}}{\partial x_{j}}
\end{array}\right)=0 \\
\frac{\partial \rho W}{\partial t}+\nabla \cdot(\underset{r}{\rho V W})=\Gamma \\
\frac{\partial \rho \varepsilon}{\partial t}+\nabla \cdot(\stackrel{\mathrm{r}}{\rho V \varepsilon})=\rho I
\end{gathered}
$$

in which, $V$ is the flow velocity. Subscript $i$ and $j$ denote the tensor notation. $\rho$ and $H$ are the density and the entropy values of the mixture phase. $W$ and $\varepsilon$ are the wetness fraction and the number of droplets per unit volume. $\Gamma$ and $I$ are the liquid mass generation rate and the nucleation rate respectively. The NEC model is applied to describe the wet steam properties based on the nucleation theory and the droplet growth model. The nucleation rate $I$ and the non-isothermal correction factor $\theta$ are used to calculate the droplet formation.

$$
\begin{gathered}
I=\frac{q_{c}}{(1+\theta)}\left(\frac{\rho_{v}^{2}}{\rho_{l}}\right) \sqrt{\frac{2 \sigma}{M_{m}^{3} \pi}} \exp \left(-\frac{4 \pi r_{*}^{2} \sigma}{3 K_{b} T}\right) \\
\theta=2 \frac{(\gamma-1)}{(\gamma+1)}\left(\frac{h_{l v}}{R T}\right)\left(\frac{h_{l v}}{R T}-0.5\right)
\end{gathered}
$$

where $q_{c}$ is the evaporation coefficient, $k_{b}$ is the Boltzmann constant, $M_{m}$ is the mass of one molecule, $\sigma$ is the liquid surface tension, $\rho_{l}$ is the liquid density, $h_{l v}$ is the specific enthalpy of evaporation at pressure $p$ and $\gamma$ is the ratio of specific heat capacities. The droplet growth is modelled as given by

$$
\begin{gathered}
\Gamma=\frac{4}{3} \pi \rho_{l} I r_{*}^{3}+4 \pi \rho_{l} \varepsilon \bar{r}^{2} \frac{\partial \bar{r}}{\partial t} \\
\frac{\partial \bar{r}}{\partial t}=\frac{p}{h_{l v} \rho_{l} \sqrt{2 \pi R T}} \frac{\gamma+1}{2 \gamma} C_{P}\left(T_{l}-T_{v}\right)
\end{gathered}
$$

where $r^{*}$ is the Kelvin-Helmholtz critical droplet radius. 
Besides, the steam properties are based on the IAPWS standard. The NEC model is coupled with $k-\omega$ shear stress transport (SST) turbulence model, which is suitable for the turbo-machinery applications. The above models have been wildly validated against the experimental data from the nozzle flows [7] and the cascade flows [8] as well as the turbine flows [9].

\section{Results and discussion}

The last stage rotor blades of a wet steam turbine run at 1500rpm are taken for the investigated case. In which the calculation domain can be minimized as one blade passage using periodic boundary condition. In order to improve the calculation accuracy, the H-O-H structured grids are used as shown in Figure 1. ANSYS CFX is employed to solve the Reynolds-averaged Navier-Stokes equations and the $k-\omega$ SST turbulence model. The boundary conditions are given in table 1 . The rotor blade is meshed use element soild185 in ANSYS Workbench. The material and geometric properties of the blade are given in table 2 .

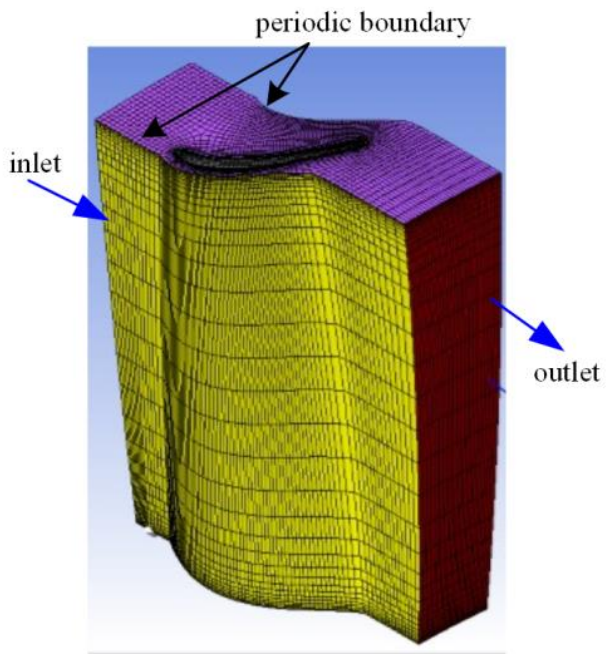

Figure 1. Calculation domain using periodic boundary condition

Table 1. Boundary conditions

\begin{tabular}{|c|c|c|}
\hline Inlet total pressure & Inlet total temperature & Pressure ratio \\
\hline $39.597 \mathrm{kPa}$ & $300 \mathrm{~K}$ & 1.71 \\
\hline
\end{tabular}

Table 2. Material and geometric properties of the blade

\begin{tabular}{|c|c|c|c|}
\hline Elasticity modulus & Density & Poisson's ratio & Length \\
\hline $198 \mathrm{GPa}$ & $7750 \mathrm{~kg} / \mathrm{m}^{3}$ & 0.3 & $1200 \mathrm{~mm}$ \\
\hline
\end{tabular}

The pressure distribution and its induced stress of the rotating blade at different intake angles $\left(A_{\text {in }}=0^{\circ}, 15^{\circ}, 30^{\circ}\right)$ and wetness fractions $\left(W_{\text {in }}=0.03,0.04,0.05\right)$ are simulated and calculated in ANSYS CFX and ANSYS Workbench respectively, as shown in Figures 2-5.

Figure 2 shows the pressure distribution on $50 \%$ blade span at different wetness fractions when the inlet angle is $0^{\circ}$. It can be found that the maximum of pressure is slightly increased with the wetness fractions. As a result, the maximum of von-Mises stress of the rotating blade is increased with the wetness fractions, as shown in Figure 3. Note that when the wetness fraction increased from 0.03 to 
0.04 , the von-Mises increased by $40.25 \%$, while when the wetness fraction increases from 0.04 to 0.05 , the von-Mises only increase by $0.54 \%$.

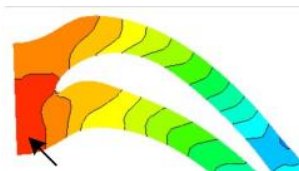

Max:3.951 kPa

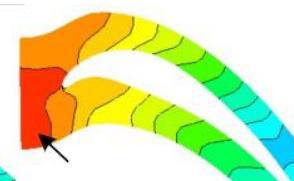

Max:4.023 kPa

(a) $A_{\text {in }}=15^{\circ}, W_{\text {in }}=0.03$

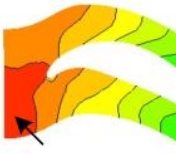

Max:4.151 kPa

(c) $A_{\text {in }}=30^{\circ}, W_{\text {in }}=0.03$

Figure 2. Comparison of the pressure distribution on the $50 \%$ blade span of three different wetness fractions.

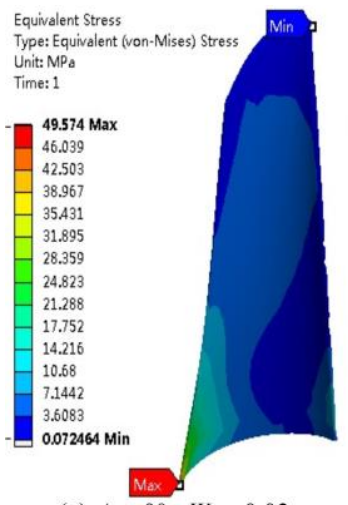

(a) $A_{\text {in }}=0^{\circ}, W_{\text {in }}=0.03$

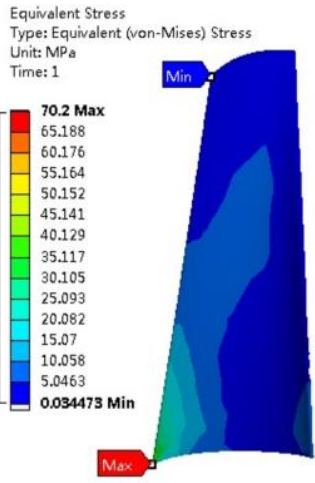

(b) $A_{\text {in }}=15^{\circ}, W_{\text {in }}=0.03$

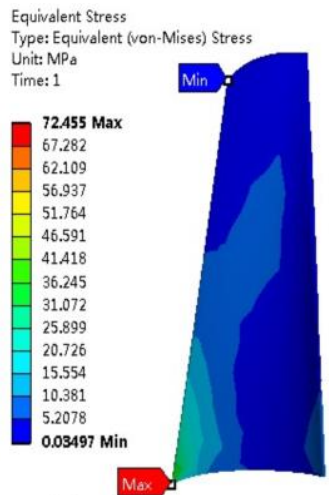

(c) $A_{\text {in }}=30^{\circ}, W_{\text {in }}=0.03$

Figure 3. Comparison of the von-Mises stress on the surface of blade of three different wetness fractions.

Figure 4 shows the pressure distribution on $50 \%$ blade span at different inlet angles when the wetness fraction is 0.03 . It can be found that the maximum of pressure is increased with the inlet angle. As a result, the maximum of von-Mises stress of the rotating blade is increased with the inlet angle, as shown in Figure 5. Note that when the inlet angle increased from $0^{\circ}$ to $15^{\circ}$, the von-Mises increased by $41.61 \%$, while when the wetness fraction increases from 0.04 to 0.05 , the von-Mises only increase by $0.36 \%$.

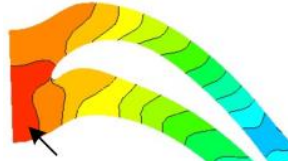

Max:3.951 kPa

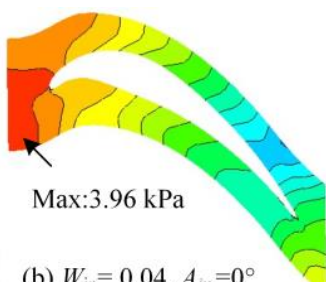

(b) $W_{\text {in }}=0.04, A_{\text {in }}=0^{\circ}$

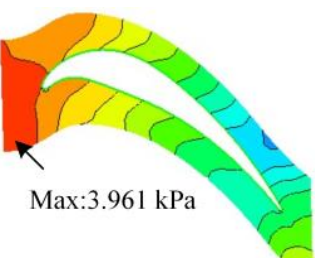

(c) $W_{\text {in }}=0.05, A_{\text {in }}=0^{\circ}$

Figure 4. Comparison of the pressure distribution on the $50 \%$ blade span of three different inlet angles. 


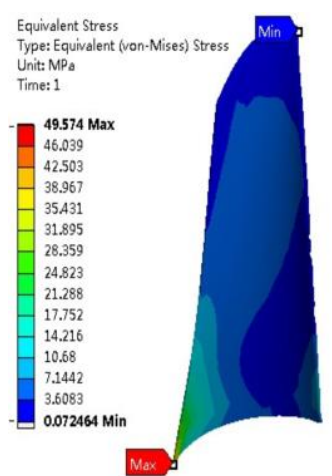

(a) $W_{\mathrm{in}}=0.03, A_{\text {in }}=0^{\circ}$

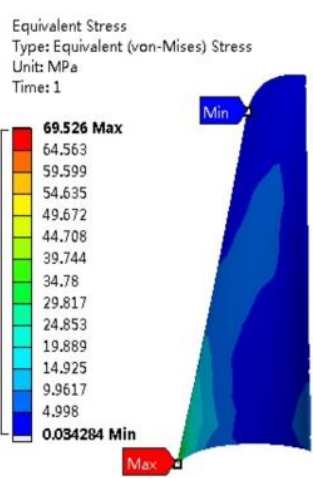

(b) $W_{\text {in }}=0.04, A_{\text {in }}=0^{\circ}$

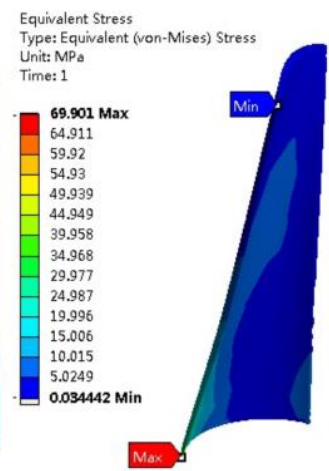

(c) $W_{\text {in }}=0.05, A_{\text {in }}=0^{\circ}$

Figure 5. Comparison of the von-Mises stress on the surface of blade of three different inlet angles.

The above results show that the higher inlet wetness fraction and/or bigger inlet angle would induce lager von-Mises stress of the blade. The maximum stress points are located in the trailing edge of the blade root, where should be to pay attention in the design.

\section{Conclusions}

This paper proposes a method to examine the influences of inlet wetness fraction and inlet angle on the strength performance of the rotating blade considering the effects of wet steam NEC. A wildly verified NEC model was employed to numerically simulate the wet steam flow with ANSYS CFX to obtain the pressure distribution on the blade surface. Applying the NEC-induced excitation, the strength analysis was carried out with ANSYS Workbench. Several conclusions were drawn as follows:

(1) Considering the effects of wet steam NEC, the von-Mises stress of the rotor blade would increase with inlet wetness fraction and let angle.

(2) The sensitive range of studied inlet condition is located at $A_{\text {in }} \in[0,15], W_{\text {in }} \in[0.03,0.04]$, in which the increases of von-Mises stress are over $40 \%$. It could be inferred that the stress of blade working in wet steam might be reduced by adjusting the inlet conditions.

(3) The presented method to calculate fluid excitation generated from wet steam NEC and its resulting stress of the blade could be extended into the design and analysis of wet steam turbines to meet the needs of engineering.

\section{Acknowledgment}

This work was supported by the key research and development program of Shandong Province (Grant No. 2017GGX40116)

\section{Nomenclature}

$A$

$C_{p}$

$H$

$h_{l v}$

I

$k_{b}$

$L$

$p$
Inlet angle, specific heat, $\mathrm{J} /(\mathrm{kg} \mathrm{K})$ total enthalpy of mixture phase, $\mathrm{J} / \mathrm{kg}$ specific enthalpy of evaporation, $\mathrm{J} / \mathrm{kg}$ nucleation rate Boltzmann constant $=1.3807 \times 10^{-23}, \mathrm{~J} / \mathrm{K}$ blade height, $\mathrm{m}$ pressure, $\mathrm{Pa}$
Kelvin-Helmholtz critical droplet radius, $m$ super saturation ratio $=p / p_{\text {sat }}(T)$ temperature of mixture phase, $\mathrm{K}$ flow velocity, $\mathrm{m} / \mathrm{s}$ wetness fraction vapor specific heat ratio liquid mass generation rate number of droplets per unit volume $=$ 


$$
\begin{gathered}
p_{\text {sat }}(T) \\
q_{c} \\
r \\
R \\
R_{d}
\end{gathered}
$$

$$
\begin{gathered}
\text { equilibrium saturation pressure, } \mathrm{Pa} \\
\text { condensation coefficient } \\
\text { droplet radius, } \mathrm{m} \\
\text { gas specific constant, } \mathrm{J} /(\mathrm{mol} \mathrm{K}) \\
\text { radius of shaft, } \mathrm{m}
\end{gathered}
$$

$$
\beta \rho_{g} /(1-\beta) V_{d} \rho_{l}
$$

non-isothermal correction factor effective thermal conductivity, $\mathrm{W} /(\mathrm{m} \mathrm{K})$ effective dynamic viscosity, $\mathrm{kg} /(\mathrm{m} \mathrm{s})$ density of mixture phase $=\rho_{g} /(1-\beta), \mathrm{kg} / \mathrm{m}^{3}$ liquid surface tension, $\mathrm{N} / \mathrm{m}$

\section{References}

1. Lau, Y. L., Leung, R. C. K., So, R. M. C. Vortex-induced vibration effect on fatigue life estimate of turbine blades. Journal of Sound and Vibration 307, 698-719 (2007)

2. García, J., Kubiak, J., Sierra, F., Urquiza, G., Rodríguez, J. Numerical analysis of the blade forces caused by wake/blade interaction in the last stage of a steam turbine. Proceedings of power, (St. Antonio, Texas, 2007)

3. Ford, I. Statistical mechanics of nucleation: a review. P I Mech Eng C-J Mec, 218, 883-899 (2004)

4. Young, J. B. Two-dimensional nonequilibrium, wet-steam calculations for nozzles and turbine cascades. J Turbomach, 114, 569-579 (1992)

5. Young, J. B. The spontaneous condensation od steam in supersonic nozzles. Physicochem Hydrodyn, 3, 57-82 (1982)

6. K. Ishazaki, T. I., Daiguji, H. A High-Resolution Numerical Method for Transonic Nonequilibrium Condensation Flows Through a Steam Turbine Cascade. J. Turbomach, 1, 479-484. (1995)

7. Moses, C. A., Stein, G. D. On the growth of steam droplets formed in a Laval nozzle using both static pressure and light scattering measurements. J Fluid Eng, 100, 311-322 (1978)

8. White, A. J., Walters, P. T. Experimental validation of condensing flow theory for a stationary cascade of steam turbine blades. P. Roy. Soc. A-Math. Phy. 354, 59-88 (1996)

9. Gardzilewicz, A., Kolovratnik, M. Numerical and experimental investigations of steam condensation in LP part of a large power turbine. J. of Fluids Eng., 131, 041301(2009) 